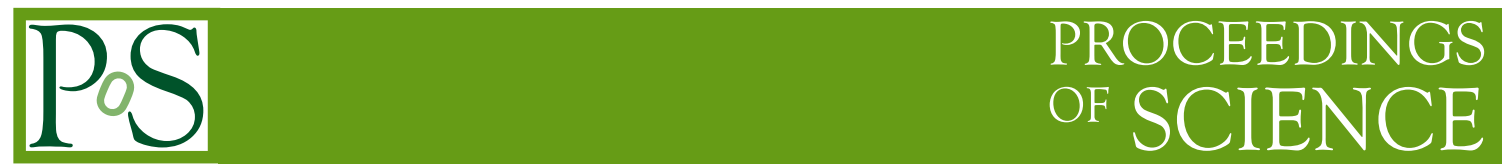

\title{
QCD vacuum energy in 5 loops
}

\section{P. A. Baikov,}

Skobeltsyn Institute of Nuclear Physics, Lomonosov Moscow State University, 1(2),

Leninskie gory, Moscow 119991, Russian Federation

E-mail: baikovatheory.sinp.msu.ru

\section{K. G. Chetyrkin*,}

II. Institut für Theoretische Physik Universität Hamburg Luruper Chaussee 149,

22761 Hamburg, Germany

E-mail : konstantin. chetyrkin@desy. de

We report about analytic calculation of the five-loop contribution to the anomalous dimension of the QCD vacuum energy. The result completes recent series of works [1-7] devoted to the renormalization of QCD at the five-loop level.

13th International Symposium on Radiative Corrections (Applications of Quantum Field Theory to Phenomenology)

25-29 September, 2017

St. Gilgen, Austria

${ }^{*}$ Speaker. 


\section{Introduction}

Last year saw a remarkable development in the program of renormalization of the QCD Lagrangian: the $\beta$-function, the quark anomalous dimension as well as anomalous dimensions of all relevant quantum fields had been computed at the five-loop level [2-7] for the case of a generic gauge group. Essentially every result has been successfully cross-checked by (at least) two independent calculations! It is worthwhile to note, that at the 4-loop level it took almost 8 years before the pioneering result for the QCD $\beta$-function [8] was independently confirmed [9].

In our talk we describe analytic calculation of the five-loop contribution to the anomalous dimension of the QCD vacuum energy. The result provides the last missing piece for the complete renormalization of the QCD Lagrangian in 5 loops.

\section{Preliminaries}

Our starting point is the QCD Lagrangian with $n_{f}$ quark flavors written in terms of (bare) fields, coupling constant $g_{B}$ and quark masses $m_{f}^{B}$ :

$$
\mathscr{L}_{Q C D}^{\text {full }}=-\frac{1}{4}\left(G_{\mu \nu}^{B}\right)^{2}+\bar{\psi}_{B}\left(i \hat{\mathscr{D}}-\mathbf{m}^{B}\right) \psi_{B}-E_{0}^{B}
$$

where the $\mathbf{m}^{B}$ stands for a (diagonal) matrix of the bare quark masses $m_{f}^{B}$ (with $f$ running from 1 to $n_{f}$ ). The bare coupling constant, quark masses, gluon, quark and ghost fields are related to the renormalized ones as follows:

$$
g^{B}=\sqrt{Z_{g}} g, m_{f}^{B}=Z_{m} m_{f}, A_{0}^{a \mu}=\sqrt{Z_{3}} A^{a \mu}, \quad \psi_{0}^{f}=\sqrt{Z_{2}} \psi^{f}, c_{0}^{a}=\sqrt{Z_{3}^{c}} c^{a} .
$$

$E_{0}(\mu)$ is the renormalized (density of) vacuum energy

$$
E_{0}^{B}=\mu^{-2 \varepsilon}\left(E_{0}(\mu)-Z_{0}^{\mathrm{di}} \sum_{f} m_{f}^{4}-Z_{0}^{\mathrm{nd}} \sum_{f, f^{\prime}}^{f \neq f^{\prime}} m_{f}^{2} m_{f^{\prime}}^{2}\right), \quad \varepsilon=(4-D) / 2,
$$

while $Z_{0}^{\text {di }}$ and $Z_{0}^{\text {nd }}$ are the corresponding renormalization constants (RCs) [10].

In terms of the renormalized fields the Lagrangian reads ${ }^{1}$.

$$
\begin{aligned}
\mathscr{L}_{Q C D}^{\text {full }} & =-\frac{1}{4} Z_{3}\left(\partial_{\mu} A_{v}-\partial_{v} A_{\mu}\right)^{2}-\frac{1}{2} g Z_{1}^{3 g}\left(\partial_{\mu} A_{v}^{a}-\partial_{v} A_{\mu}^{a}\right)\left(A_{\mu} \times A_{v}\right)^{a} \\
& -\frac{1}{4} g^{2} Z_{1}^{4 g}\left(A_{\mu} \times A_{v}\right)^{2}-\frac{1}{2 \xi_{L}}\left(\partial_{v} A_{\mu}\right)^{2}+Z_{3}^{c} \partial_{\nu} \bar{c}\left(\partial_{\nu} c\right)+g Z_{1}^{c c g} \partial^{\mu} \bar{c}\left(A_{\mu} \times c\right) \\
& +Z_{2} \sum_{f=1}^{n_{f}} \bar{\psi}^{f} \mathrm{i} \partial \psi^{f}+g Z_{1}^{\psi \psi g} \sum_{f=1}^{n_{f}} \bar{\psi}^{f} A \psi^{f}-Z_{\psi \psi} \sum_{f=1}^{n_{f}} m_{f} \bar{\psi}^{f} \psi^{f}-E_{0}^{B} .
\end{aligned}
$$

All the RCs appearing in (2.4) have been recently computed at 5 loops except for $Z_{0}^{\mathrm{di}}$ and $Z_{0}^{\text {nd }}$. The latter are (partially) known at 4 loops (see [11] and references therein).

\footnotetext{
${ }^{1}$ For simplicity we set the $\mathrm{t}^{\prime}$ Hooft mass $\mu=1$ in eqs. (2.1), 2.2 and (2.4).
} 
The anomalous dimension of the renormalized vacuum energy reads:

$$
\begin{aligned}
\hat{\gamma}_{0}(m) & \equiv \mu^{2} \frac{d}{d \mu^{2}} E_{0}=\left(4 \gamma_{m}-\varepsilon\right) \hat{Z}_{0}(m)+(-\varepsilon+\beta) a_{s} \frac{\partial}{\partial a_{s}} \hat{Z}_{0}(m) \\
& =\left(\sum_{f} m_{f}^{4}\right) \gamma_{0}^{\mathrm{d} i}\left(a_{s}\right)+\left(\sum_{f, f^{\prime}}^{f \neq f^{\prime}} m_{f}^{2} m_{f^{\prime}}^{2}\right) \gamma_{0}^{\mathrm{nd}}\left(a_{s}\right),
\end{aligned}
$$

where $a_{s}=\frac{g^{2}}{4 \pi^{2}}=\frac{\alpha_{s}}{\pi}$ and

$$
\hat{Z}_{0}(m) \equiv Z_{0}^{\mathrm{di}} \sum_{f} m_{f}^{4}+Z_{0}^{\mathrm{nd}} \sum_{f, f^{\prime}}^{f \neq f^{\prime}} m_{f}^{2} m_{f^{\prime}}^{2} .
$$

For the case of just one massive quark we have $\hat{\gamma}_{0}=m_{q}^{4} \gamma_{0}^{\mathrm{d} i}\left(a_{s}\right)$ and $\gamma_{0}^{\text {nd }} \equiv 0$.

\section{Calculation and results}

We have computed the 5-loop contribution to the $\hat{\gamma}_{0}$ within the massless approach (that is with the use of the global $R^{*}$-operation [12-14], the FORM $[15,16]$ program BAICER [17] and the computer facilities of the KIT) for a general case of arbitrary many quark flavors with different masses. Our results for

$$
\gamma_{0}^{\mathrm{di}}=\sum_{i \geq 0}\left(\gamma_{0}^{\mathrm{di}}\right)_{i}\left(\frac{\alpha_{s}}{4 \pi}\right)^{i} \text { and } \gamma_{0}^{\mathrm{nd}}=\sum_{i \geq 2}\left(\gamma_{0}^{\mathrm{nd}}\right)_{i}\left(\frac{\alpha_{s}}{4 \pi}\right)^{i}
$$

read

$$
\begin{gathered}
\left(\gamma_{0}^{\mathrm{di}}\right)_{0}=\{-d R\}, \quad\left(\gamma_{0}^{\mathrm{di}}\right)_{1}=\frac{d R}{16 \pi^{2}}\left\{-4 C_{F}\right\} \\
\left(\gamma_{0}^{\mathrm{di}}\right)_{2}=\frac{d R}{16 \pi^{2}}\left\{C_{F}^{2}\left[\frac{131}{2}-48 \zeta_{3}\right]+C_{F} C_{A}\left[-\frac{109}{2}+24 \zeta_{3}\right]+10 C_{F} T_{f} n_{f}+48 C_{F} T_{f}\right\}, \\
\left(\gamma_{0}^{\mathrm{di}}\right)_{3}=\frac{d R}{16 \pi^{2}}\left\{C_{F}^{3}\left[-\frac{2942}{3}+48 \zeta_{3}+288 \zeta_{4}+160 \zeta_{5}\right]+C_{F} T_{f}^{2} n_{f}^{2}\left[\frac{10912}{243}-\frac{128}{3} \zeta_{3}\right]\right. \\
+C_{F} T_{f}^{2} n_{f}\left[-\frac{256}{9}\right]+C_{F}^{2} T_{f} n_{f}\left[\frac{562}{3}+\frac{32}{3} \zeta_{3}-160 \zeta_{4}\right] \\
+C_{F} T_{f} n_{f} C_{A}\left[-\frac{2644}{243}+16 \zeta_{3}+128 \zeta_{4}\right]+C_{F}^{2} T_{f}[-64] \\
+C_{F} T_{f} C_{A}\left[\frac{5888}{9}+352 \zeta_{3}-160 \zeta_{5}\right]+C_{F}^{2} C_{A}\left[\frac{3584}{3}-\frac{3304}{3} \zeta_{3}+32 \zeta_{4}+720 \zeta_{5}\right] \\
\left.+C_{F} C_{A}^{2}\left[-\frac{121547}{243}+\frac{1880}{3} \zeta_{3}-88 \zeta_{4}-520 \zeta_{5}\right]\right\}
\end{gathered}
$$




$$
\begin{aligned}
& \left(\gamma_{0}^{\mathrm{di}}\right)_{4}=\frac{d R}{16 \pi^{2}}\left\{C_{F}^{4}\left[\frac{787555}{48}-9470 \zeta_{3}+5568 \zeta_{3}^{2}+432 \zeta_{4}-5232 \zeta_{5}-1200 \zeta_{6}-3024 \zeta_{7}\right]\right. \\
& +C_{F} T_{f}^{3} n_{f}^{3}\left[\frac{1492}{27}+\frac{832}{27} \zeta_{3}-\frac{256}{3} \zeta_{4}\right]+C_{F} T_{f}^{3} n_{f}^{2}\left[-\frac{256}{9}\right] \\
& +C_{F}^{2} T_{f}^{2} n_{f}^{2}\left[-\frac{180253}{243}+\frac{3376}{9} \zeta_{3}+448 \zeta_{4}-\frac{1024}{3} \zeta_{5}\right]+C_{F}^{2} T_{f}^{2} n_{f}\left[-\frac{18176}{9}+1920 \zeta_{3}\right] \\
& +C_{F} T_{f}^{2} n_{f}^{2} C_{A}\left[\frac{103547}{486}-\frac{10064}{9} \zeta_{3}-40 \zeta_{4}+\frac{2816}{3} \zeta_{5}\right] \\
& +C_{F} T_{f}^{2} n_{f} C_{A}\left[-\frac{14080}{9}-\frac{8704}{3} \zeta_{3}-96 \zeta_{3}^{2}+528 \zeta_{4}+640 \zeta_{5}-400 \zeta_{6}\right] \\
& +C_{F}^{3} T_{f} n_{f}\left[-\frac{27373}{18}-\frac{3244}{3} \zeta_{3}-1024 \zeta_{3}^{2}-1700 \zeta_{4}+4624 \zeta_{5}+1600 \zeta_{6}\right] \\
& +C_{F}^{3} T_{f}\left[2360+3136 \zeta_{3}-4480 \zeta_{5}\right] \\
& +C_{F}^{2} C_{A} T_{f} n_{f}\left[\frac{1388131}{972}+\frac{13004}{9} \zeta_{3}+2176 \zeta_{3}^{2}-2000 \zeta_{4}-\frac{17512}{3} \zeta_{5}+1600 \zeta_{6}\right] \\
& +C_{F}^{2} C_{A} T_{f}\left[-\frac{31160}{9}+8544 \zeta_{3}+480 \zeta_{3}^{2}-1584 \zeta_{4}-9920 \zeta_{5}+1200 \zeta_{6}+2240 \zeta_{7}\right] \\
& +C_{F} T_{f} n_{f} C_{A}^{2}\left[\frac{744661}{486}+\frac{466}{9} \zeta_{3}-1032 \zeta_{3}^{2}+2376 \zeta_{4}+\frac{5444}{3} \zeta_{5}-2300 \zeta_{6}-294 \zeta_{7}\right] \\
& +C_{F} T_{f} C_{A}^{2}\left[9456+2896 \zeta_{3}+584 \zeta_{3}^{2}-1452 \zeta_{4}-\frac{17360}{3} \zeta_{5}+1100 \zeta_{6}+\frac{10997}{3} \zeta_{7}\right] \\
& +C_{F}^{3} C_{A}\left[-\frac{287954}{9}+\frac{22844}{3} \zeta_{3}-6088 \zeta_{3}^{2}+5764 \zeta_{4}+27296 \zeta_{5}-6500 \zeta_{6}-9184 \zeta_{7}\right] \\
& +C_{F}^{2} C_{A}^{2}\left[\frac{23242925}{972}-\frac{70940}{9} \zeta_{3}-1820 \zeta_{3}^{2}+1719 \zeta_{4}-14402 \zeta_{5}-2150 \zeta_{6}+14182 \zeta_{7}\right] \\
& +C_{F} C_{A}^{3}\left[-\frac{28785743}{3888}+\frac{167719}{27} \zeta_{3}+2318 \zeta_{3}^{2}-\frac{9280}{3} \zeta_{4}-\frac{17512}{3} \zeta_{5}+4675 \zeta_{6}-\frac{14525}{6} \zeta_{7}\right] \\
& +n_{f} \frac{d_{F}^{a b c d} d_{F}^{a b c d}}{d_{R}}\left[-824-5584 \zeta_{3}+2304 \zeta_{3}^{2}+720 \zeta_{4}-3680 \zeta_{5}+7056 \zeta_{7}\right] \\
& +\frac{d_{F}^{a b c d} d_{F}^{a b c d}}{d_{R}}\left[-2816 \zeta_{3}+2688 \zeta_{3}^{2}+8320 \zeta_{5}-5320 \zeta_{7}\right] \\
& \left.+\frac{d_{F}^{a b c d} d_{A}^{a b c d}}{d_{R}}\left[352+3608 \zeta_{3}-11136 \zeta_{3}^{2}-360 \zeta_{4}+2492 \zeta_{7}\right]\right\},
\end{aligned}
$$$$
\left(\gamma_{0}^{\mathrm{nd}}\right)_{2}=\frac{d R}{16 \pi^{2}}\left\{48 C_{F} T_{f}\right\}
$$$$
\left(\gamma_{0}^{\mathrm{nd}}\right)_{3}=\frac{d R}{16 \pi^{2}}\left\{C_{F} T_{f}^{2} n_{f}\left[-\frac{256}{9}\right]+C_{F}^{2} T_{f}[-64]+C_{F} T_{f} C_{A}\left[\frac{5888}{9}+352 \zeta_{3}-160 \zeta_{5}\right]\right\}
$$ 


$$
\begin{aligned}
\left(\gamma_{0}^{\mathrm{nd}}\right)_{4} & =\frac{d R}{16 \pi^{2}}\left\{C_{F} T_{f}^{3} n_{f}^{2}\left[-\frac{256}{9}\right]+C_{F}^{2} T_{f}^{2} n_{f}\left[-\frac{18176}{9}+1920 \zeta_{3}\right]\right. \\
& +C_{F} T_{f}^{2} n_{f} C_{A}\left[-\frac{14080}{9}-\frac{8704}{3} \zeta_{3}-96 \zeta_{3}^{2}+528 \zeta_{4}+640 \zeta_{5}-400 \zeta_{6}\right] \\
& +C_{F}^{3} T_{f}\left[2360+3136 \zeta_{3}-4480 \zeta_{5}\right] \\
& +C_{F}^{2} C_{A} T_{f}\left[-\frac{31160}{9}+8544 \zeta_{3}+480 \zeta_{3}^{2}-1584 \zeta_{4}-9920 \zeta_{5}+1200 \zeta_{6}+2240 \zeta_{7}\right] \\
& +C_{F} T_{f} C_{A}^{2}\left[9456+2896 \zeta_{3}+584 \zeta_{3}^{2}-1452 \zeta_{4}-\frac{17360}{3} \zeta_{5}+1100 \zeta_{6}+\frac{10997}{3} \zeta_{7}\right] \\
& \left.+\frac{d_{F}^{a b c d} d_{F}^{a b c d}}{d_{R}}\left[-2816 \zeta_{3}+2688 \zeta_{3}^{2}+8320 \zeta_{5}-5320 \zeta_{7}\right]\right\} .
\end{aligned}
$$

Here $\zeta$ is the Riemann zeta-function ( with $\zeta_{3}=1.2020569 \ldots, \zeta_{4}=1.0823232 \ldots, \zeta_{5}=1.0369278 \ldots$, $\left.\zeta_{6}=1.0173431 \ldots, \zeta_{7}=1.0083493 \ldots\right) . C_{F}$ and $C_{A}$ are the quadratic Casimir operators of the quark $\left[T^{a} T^{a}\right]_{i j}=C_{F} \delta_{i j}$ and the adjoint $\left[C^{a} C^{a}\right]_{b d}=C_{A} \delta_{b d},\left(C^{a}\right)_{b c}=-i f^{a b c}$ representations of the Lie algebra. $n_{f}$ stands for the number of quark flavors, $d_{R}$ is dimension of the quark representation of the gauge group and $T_{f}$ refers to the trace normalization $\operatorname{tr}\left(T^{a} T^{b}\right)=T_{f} \delta^{a b}$. The higher order group invariants are defined according to $[8,18]$.

Note that if a color structure contributes to $\gamma_{0}^{\text {nd }}$ then the same color structure also appears in $\gamma_{0}^{\mathrm{di}}$ with an identical coefficient. This is a direct consequence of our way of separating $\hat{\gamma}_{0}(m)$ into two pieces in eq. (2.5).

For the QCD with the colour group SU(3) we have

$$
\begin{aligned}
& \gamma_{0}^{\mathrm{di}}=\frac{1}{16 \pi^{2}}\{-3-4 \frac{\alpha_{s}}{\pi}+\left(\frac{\alpha_{s}}{\pi}\right)^{2}\left[-\frac{313}{24}+\frac{5}{4} n_{f}+2 \zeta_{3}\right] \\
&+\left(\frac{\alpha_{s}}{\pi}\right)^{3}[-\frac{14251}{432}+\left(\frac{341}{486}-\frac{2}{3} \zeta_{3}\right) n_{f}^{2}+\frac{231}{2} \zeta_{3} \\
&\left.+n_{f}\left(\frac{4109}{648}+\frac{35}{18} \zeta_{3}+\frac{16}{3} \zeta_{4}\right)-\frac{19}{2} \zeta_{4}-\frac{1975}{18} \zeta_{5}\right] \\
&+\left(\frac{\alpha_{s}}{\pi}\right)^{4}[-\frac{303061}{3072}+\frac{882061}{432} \zeta_{3}+\frac{20083}{288} \zeta_{3}^{2}-\frac{124511}{192} \zeta_{4} \\
&-\frac{11543}{3} \zeta_{5}+\frac{632375}{576} \zeta_{6}+\frac{36883}{32} \zeta_{7} \\
&+ n_{f}\left(\frac{6286061}{62208}+\frac{593}{864} \zeta_{3}-\frac{2327}{144} \zeta_{3}^{2}+\frac{50867}{576} \zeta_{4}+\frac{1571}{144} \zeta_{5}-\frac{27125}{288} \zeta_{6}-\frac{147}{16} \zeta_{7}\right) \\
&+\left.\left.n_{f}^{2}\left(-\frac{530837}{373248}-\frac{4817}{432} \zeta_{3}+\frac{179}{96} \zeta_{4}+\frac{83}{9} \zeta_{5}\right)+n_{f}^{3}\left(\frac{373}{3456}+\frac{13}{216} \zeta_{3}-\frac{1}{6} \zeta_{4}\right)\right]\right\}, \\
& \gamma_{0}^{\text {nd }}=\frac{1}{16 \pi^{2}}\left\{6\left(\frac{\alpha_{s}}{\pi}\right)^{2}+\left(\frac{\alpha_{s}}{\pi}\right)^{3}\left[\frac{176}{3}+33 \zeta_{3}-15 \zeta_{5}+n_{f}\left(-\frac{4}{9}\right)\right]\right. \\
&+\left(\frac{\alpha_{s}}{\pi}\right)^{4}\left[\left(\frac{14147}{24}+\frac{36691}{72} \zeta_{3}+\frac{967}{16} \zeta_{3}^{2}-\frac{4851}{32} \zeta_{4}-\frac{6890}{9} \zeta_{5}+\frac{3675}{32} \zeta_{6}+\frac{3829}{12} \zeta_{7}\right)\right. \\
&\left.\left.\left.\quad+n_{f}\left(-\frac{779}{27}-24 \zeta_{3}-\frac{9}{8} \zeta_{3}^{2}+\frac{99}{16} \zeta_{4}+\frac{15}{2} \zeta_{5}-\frac{75}{16} \zeta_{6}\right)-\frac{1}{18} n_{f}^{2}\right)\right]\right\}
\end{aligned}
$$


or, numerically,

$$
\begin{aligned}
& \gamma_{0}^{\mathrm{di}}=\frac{-3}{16 \pi^{2}}\left(1+1.333 a_{s}+\left(3.546-0.4167 n_{f}\right) a_{s}^{2}\right. \\
& \left(6.069-4.8170 n_{f}+0.03327 n_{f}^{2}\right) a_{s}^{3} \\
& \left.+\left(-14.658-26.779 n_{f}+1.0816 n_{f}^{2}+0.000038 n_{f}^{3}\right) a_{s}^{4}\right) \\
& =1 .+1.3333 a_{s}+1.04585 a_{s}^{2}-21.636 a_{s}^{3}-136.384 a_{s}^{4} \text { if } n_{f}=6 \\
& \gamma_{0}^{\mathrm{nd}}=\frac{3}{8 \pi^{2}}\left(a_{s}^{2}+\left(13.7968-0.07407 n_{f}\right) a_{s}^{3}+\left(128.339-8.2703 n_{f}-0.0093 n_{f}^{2}\right) a_{s}^{4}\right)
\end{aligned}
$$

\section{Applications}

\subsection{Mixing of all scalar operators of dimension 4}

Along with the $\beta$-function and quark mass anomalous dimension $\gamma_{m}$ the vaccum anomalous dimension $\hat{\gamma}_{0}$ lead to a complete description of the renormalization mixing of the operators

$$
O_{1}=G_{\mu \nu}^{a} G_{\mu \nu}^{a}, \quad O_{2}^{i j}=m_{i} \bar{\psi}_{j} \psi_{j}, O_{6}^{i j}=m_{i}^{2} m_{j}^{2} .
$$

The corresponding matrix of anomalous dimensions reads $[10,19,20]$

$$
\begin{gathered}
\mu^{2} \frac{d}{d \mu^{2}} O_{1}=-\left(a_{s} \frac{\partial}{\partial a_{s}} \beta\right) O_{1}+4\left(a_{s} \frac{\partial}{\partial a_{s}} \gamma_{m}\right) \sum_{i} O_{2}^{i i}+4 a \frac{\partial}{\partial a_{s}} \hat{g}_{0}, \\
\mu^{2} \frac{d}{d \mu^{2}} O_{2}^{i j}=-m_{i} \frac{\partial}{\partial m_{j}} \hat{g}_{0}, \\
\mu^{2} \frac{d}{d \mu^{2}} O_{6}^{i j}=4 \gamma_{m} O_{6}^{i j} .
\end{gathered}
$$

Here

$$
\mu^{2} \frac{d}{d \mu^{2}} a_{s}=a_{s} \beta\left(a_{s}\right) \text { and } \mu^{2} \frac{d}{d \mu^{2}} m_{i}=m_{i} \gamma_{m}\left(a_{s}\right) .
$$

\subsection{Quadratic mass corrections to $R(s)$}

As $\hat{\gamma}_{0}$ fully describes the mixing of $m \bar{\psi} \psi$ to $m^{4}$, our result could be effectively employed to find the quartic mass corrections to $\mathrm{R}(\mathrm{s})$ at order $\alpha_{s}^{4}$. At orders $\alpha_{s}^{2}$ and $\alpha_{s}^{3}$ it was done in [20] and [21] respectively.

\subsection{Application for the RG optimized perturbative theory}

$\hat{\gamma}_{0}$ as well as the (perturbatively computed with all quark massive) VEV $\bar{\psi} \psi$ are main ingredients of the so-called RG optimized perturbation theory [22,23] as applied to the chiral condensate (in the massless limit!). Using the previous 4-loop $\gamma_{0}$ and the 3-loop value of $\langle\bar{\psi} \psi\rangle$ the authors of [24] arrived at

$$
-<\bar{\psi} \psi>^{1 / 3}(2 \mathrm{GeV})=281 \pm 4 \pm 7 \mathrm{MeV}
$$

which is in agreement to other independent determinations. It would be useful to upgrade the analysis by one more order of PT. 


\section{Conclusions}

We have computed the QCD vacuum anomalous dimension at 5 loops and, thus, have finished the program of the 5-loop renormalization of the QCD Lagrangian.

The authors are grateful to the Institut für Theoretische Teilchenphysik of Karlsruher Institut für Technologie (KIT) for support and kind permission to use its computer facilities.

The work of P. A. Baikov was supported in part by the grant RFBR 17-02-00175A of the Russian Foundation for Basic Research. The work of K. G. Chetyrkin was supported by the German Federal Ministry for Education and Research BMBF through Grant No. 05H15GUCC1.

\section{References}

[1] P. A. Baikov, K. G. Chetyrkin and J. H. Kühn, Quark Mass and Field Anomalous Dimensions to $\mathscr{O}\left(\alpha_{s}^{5}\right)$, JHEP $10(2014) 76$, [1402.6611].

[2] P. A. Baikov, K. G. Chetyrkin and J. H. Kühn, Five-Loop Running of the QCD coupling constant, Phys. Rev. Lett. 118 (2017) 082002, [1606.08659].

[3] F. Herzog, B. Ruijl, T. Ueda, J. A. M. Vermaseren and A. Vogt, The five-loop beta function of Yang-Mills theory with fermions, JHEP 02 (2017) 090, [1 701.01404 ].

[4] T. Luthe, A. Maier, P. Marquard and Y. Schröder, Complete renormalization of QCD at five loops, JHEP 03 (2017) 020, [1 701 . 07068$].$

[5] K. G. Chetyrkin, G. Falcioni, F. Herzog and J. A. M. Vermaseren, Five-loop renormalisation of QCD in covariant gauges, 1709.08541.

[6] T. Luthe, A. Maier, P. Marquard and Y. Schröder, Five-loop quark mass and field anomalous dimensions for a general gauge group, JHEP 01 (2017) 081, [1612 . 05512].

[7] P. A. Baikov, K. G. Chetyrkin and J. H. Kühn, Five-loop fermion anomalous dimension for a general gauge group from four-loop massless propagators, JHEP 04 (2017) 119, [1702 . 01458 ].

[8] T. van Ritbergen, J. A. M. Vermaseren and S. A. Larin, The four-loop beta function in quantum chromodynamics, Phys. Lett. B400 (1997) 379-384, [hep-ph/ 9701390 ].

[9] M. Czakon, The Four-loop QCD beta-function and anomalous dimensions, Nucl.Phys. B710 (2005) 485-498, [hep-ph/0411261].

[10] V. P. Spiridonov and K. G. Chetyrkin, Nonleading mass corrections and renormalization of the operators $m \bar{\psi} \psi$ and $G_{\mu \nu}^{2}$, Sov. J. Nucl. Phys. 47 (1988) 522-527.

[11] K. G. Chetyrkin and M. F. Zoller, Leading QCD-induced four-loop contributions to the $\beta$-function of the Higgs self-coupling in the SM and vacuum stability, JHEP 06 (2016) 175, [1604 . 00853 ].

[12] K. G. Chetyrkin, Correlator of the quark scalar currents and $\Gamma_{\text {tot }}(H \rightarrow$ hadrons $)$ at $\mathscr{O}\left(\alpha_{s}^{3}\right)$ in $p Q C D$, Phys. Lett. $\mathbf{B 3 9 0}$ (1997) 309-317, [hep-ph/9608318].

[13] K. G. Chetyrkin, Corrections of order $\alpha_{s}^{3}$ to $R_{\text {had }}$ in pQCD with light gluinos, Phys. Lett. $\mathbf{B 3 9 1}$ (1997) 402-412, [hep-ph/9608480].

[14] K. G. Chetyrkin, G. Falcioni, F. Herzog and J. A. M. Vermaseren, The method of global $R^{*}$ and its applications, http://arxiv.org/abs/1801.03024v1.

[15] J. Kuipers, T. Ueda, J. Vermaseren and J. Vollinga, FORM version 4.0, Comput.Phys.Commun. 184 (2013) 1453-1467, [1203.6543]. 
[16] M. Steinhauser, T. Ueda and J. A. M. Vermaseren, Parallel versions of FORM and more, Nucl. Part. Phys. Proc. 261-262 (2015) 45-57, [1501.07119].

[17] P. A. Baikov, K. G. Chetyrkin and J. H. Kühn, Massless Propagators, R(s) and Multiloop QCD, Nucl. Part. Phys. Proc. 261-262 (2015) 3-18, [1501.06739].

[18] T. van Ritbergen, A. N. Schellekens and J. A. M. Vermaseren, Group theory factors for feynman diagrams, Int. J. Mod. Phys. A14 (1999) 41-96, [hep-ph/9802376].

[19] V. P. Spiridonov, Anomalous Dimension of $G_{\mu \nu}^{2}$ and $\beta$ Function, preprint IYaI-P-0378 (1984).

[20] K. G. Chetyrkin and J. H. Kühn, Quartic mass corrections to R(had), Nucl. Phys. B432 (1994) 337-350, [hep-ph/9406299].

[21] K. G. Chetyrkin, R. V. Harlander and J. H. Kuhn, Quartic mass corrections to $R_{\text {had }}$ at $\mathscr{O}\left(\alpha_{s}^{3}\right)$, Nucl. Phys. B586 (2000) 56-72, [hep-ph/ 0005139$].$

[22] J. L. Kneur and A. Neveu, Renormalization Group Improved Optimized Perturbation Theory: Revisiting the Mass Gap of the O(2N) Gross-Neveu Model, Phys. Rev. D81 (2010) 125012, [1004.4834].

[23] J. L. Kneur and A. Neveu, Lambda $a_{\mathrm{MS}}^{\mathrm{QCD}}$ from Renormalization Group Optimized Perturbation, Phys. Rev. D85 (2012) 014005, [1108.3501].

[24] J.-L. Kneur and A. Neveu, Chiral condensate from renormalization group optimized perturbation, Phys. Rev. D92 (2015) 074027, [1506.0750 6]. 